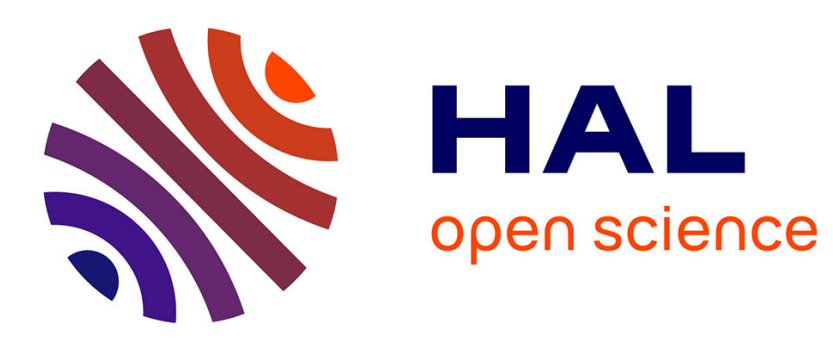

\title{
Mixture segregation by an inertial cavitation bubble
}

R. Grossier, Olivier Louisnard, Y. Vargas

\section{To cite this version:}

R. Grossier, Olivier Louisnard, Y. Vargas. Mixture segregation by an inertial cavitation bubble. Ultrasonics Sonochemistry, 2007, 14 (4), pp.431-437. 10.1016/j.ultsonch.2006.10.010 . hal-01717683

\section{HAL Id: hal-01717683 \\ https://hal.science/hal-01717683}

Submitted on 8 Nov 2018

HAL is a multi-disciplinary open access archive for the deposit and dissemination of scientific research documents, whether they are published or not. The documents may come from teaching and research institutions in France or abroad, or from public or private research centers.
L'archive ouverte pluridisciplinaire HAL, est destinée au dépôt et à la diffusion de documents scientifiques de niveau recherche, publiés ou non, émanant des établissements d'enseignement et de recherche français ou étrangers, des laboratoires publics ou privés. 


\title{
Mixture segregation by an inertial cavitation bubble.
}

\author{
R. Grossier ${ }^{\mathrm{a}}$, O. Louisnard ${ }^{\mathrm{a}, 1}$, Y. Vargas ${ }^{\mathrm{b}}$ \\ a Laboratoire de Génie des Procédés des Solides Divisés, \\ UMR CNRS 2392, École des Mines d'Albi-Carmaux, \\ Campus Jarlard, 81013 Albi Cedex 09, France \\ ${ }^{\mathrm{b}}$ Laboratorio de Ultrasonidos, USACH, Casilia 307, Santiago 2, Chile
}

\begin{abstract}
Pressure diffusion is a mass diffusion process forced by pressure gradients. It has the ability to segregate two species of a mixture, driving the densest species toward high pressure zones, but requires very large pressure gradients to become noticeable. An inertial cavitation bubble develops large pressure gradients in its vicinity, especially as the bubble rebounds at the end of its collapse, and it is therefore expected that a liquid mixture surrounding such a bubble would become segregated. Theory developed in an earlier paper shows that this is indeed the case for sufficiently large molecules or nano-particles. The main theoretical results are recalled and a possible implication of this segregation phenomenon on the well-known cavitation-enhanced crystals nucleation is proposed.
\end{abstract}

Key words: pressure diffusion, ultrasound, segregation, bubble, crystallization, cavitation,

PACS: 47.57.ef

PACS: 47.55.dd

PACS: 68.55.Ac

\section{Introduction}

The liquid surrounding a cavitation bubble is generally not pure, but is rather a mixture of two or more chemical species. For example, in sonocrystallization in solutions, the liquid is a mixture of a solvent and a dissolved species. The size of the species may vary over a wide range, from small molecules up to polymers, crystal precursors or nano-particles. All these objects are sufficiently small to undergo Brownian motion, which practical macroscopic implication is that any appearance of a concentration gradient is counteracted by mass diffusion, tending to restore equilibrium. Segregation of two species would therefore have no reason to occur, except if there is a disequilibrium between

\footnotetext{
Email address: louisnar@enstimac.fr (O. Louisnard).

1 Corresponding author
}

the species at the bubble inter-phase, for example if one species is very volatile, or in the case of a surface-active species.

In fact, several secondary diffusion mechanisms should also be considered but are generally of lesser importance. Among them, pressure diffusion is a mass-diffusion process forced by a pressure gradient, which drives the lightest species toward low pressure regions [1]. This effect is generally neglected since it requires huge pressure-gradients or very large timescales to become noticeable. It is however commonly known to produce slow gas segregation in a quiet atmosphere and is used in ultracentrifugation to separate large molecules or nano-particles from a host fluid. In fact, it also occurs in any flow involving large pressure-gradients (and therefore large accelerations). It is a purely mechanical effect and can be easily illustrated: let's consider a flowing fluid containing spherical particles heavier than 
the fluid. If the fluid flows at constant velocity, the velocity of the particles relative to the fluid will relax rapidly to zero, owing to viscous friction. Now if the fluid brakes at constant rate (which results in the appearance of a pressure gradient), the heavier particles will keep their earlier motion by inertia and acquire a positive drift velocity relative to the fluid, so that a nonzero molecular flux of particles will arise. In the case of lighter particles, the leading force is the generalized buoyancy force (sometimes referred as "Chen force" in hydrodynamics) which produces the opposite effect. The pressure diffusion flux results from the competition between inertia and generalized buoyancy and is therefore proportional to the difference between the apparent densities of the two species.

The radial motion of an inertial cavitation bubble is sufficiently violent so that one may reasonably think that pressure diffusion produces noticeable effects. Indeed, calculations of the bubble dynamics predict accelerations of the liquid near the bubble wall of the order of $10^{12} g$ [2]. In fact, pressure diffusion has been considered by Storey \& Szeri [3], along with other secondary mass-diffusion process, to study the segregation of gases inside a sonoluminescing single bubble. Here we investigate the effect of pressure diffusion on the segregation of a binary mixture surrounding a radially oscillating bubble. We report in this paper theoretical predictions from an analytical solution of the transport problem obtained in an earlier paper [4], and we propose a qualitative microscopical mechanism for sono-crystallization, consistent with our theoretical predictions.

\section{Theory}

\subsection{The convective-diffusive transport problem}

We consider a single bubble oscillating radially in a binary mixture. Both species are assumed nonvolatile and surface-inactive, so that in absence of any other segregation process, they should move together around the bubble and no concentration gradient should appear. Taking into account pressure diffusion, along with convection and classical diffusion around the oscillating bubble, the transport equation for one of a species, hereafter labeled " $\mathrm{A}$ " reads:

$$
\frac{\partial C_{A}}{\partial t}=-\boldsymbol{v} \cdot \boldsymbol{\nabla} C_{A}+D \boldsymbol{\nabla} \cdot\left[\boldsymbol{\nabla} C_{A}+\tilde{\beta} C_{A} \boldsymbol{\nabla} p\right],
$$

where, $C_{A}$ is the concentration field of species A, $\boldsymbol{v}$ and $p$ are the velocity and pressure fields respectively, $D$ is the mass diffusion coefficient of the mixture, and $\tilde{\beta}$ is the segregation parameter defined by

$$
\tilde{\beta}=\frac{M_{A}}{\mathcal{R} T}\left(\frac{\bar{V}_{A}}{M_{A}}-\frac{1}{\rho}\right) .
$$

$M_{A}$ is the molar weight of species $\mathrm{A}, \bar{V}_{A} / M_{A}$ the inverse of its apparent density and $\rho$ the average mixture density. From left to right in the right-hand side of equation (1), one can recognize respectively the convective flux, the classical Fick diffusion flux and finally the pressure diffusion flux. Since the bubble wall is assumed impermeable to species A, the total diffusive flux (the square bracket in Eq. 1) should vanish there. Besides, the mixture is considered unperturbed at time $t=0$ and far from the bubble, so that $C_{A}(\infty, t)=C_{A}(0, r)=C_{A 0}$.

\subsection{Analytical solution}

This equations set was solved analytically by perturbation methods in ref. [4] and we recall here the main results. The concentration field is obtained as the sum of two contributions, an average one and an oscillatory one. When expressed at the bubble wall, the segregation ratio $C_{A}(0, t) / C_{A 0}$ reads (we refer the reader to ref. [4] for more details):

$$
\frac{C_{A}(0, \tau)}{C_{A 0}}=\bar{c}+\Delta c(\tau)
$$

where

$$
\bar{c}=e^{\beta I}, \quad \Delta c(\tau)=-\beta e^{\beta I}\left(\frac{D}{R_{0}^{2} \omega}\right)^{1 / 2} G(\tau),
$$

and

$$
\beta=\frac{1}{2} \rho R_{0}^{2} \omega^{2} \tilde{\beta}
$$

is the dimensionless version of the segregation parameter given by $(2), R_{0}$ is the bubble ambient radius, $\omega$ the angular frequency of the bubble driving field and $\tau=\omega t$ the dimensionless time. $I$ is a bubble dynamics-dependent integral

$$
I=\int_{0}^{\infty}\left\langle\frac{4}{9} \frac{\dot{V}^{2}}{(3 \sigma+V)}\right\rangle \frac{d \sigma}{\left\langle(3 \sigma+V)^{4 / 3}\right\rangle},
$$

where notation $\langle$.$\rangle denotes average over one acoustic$ cycle, $V$ is the bubble volume non-dimensionalized 


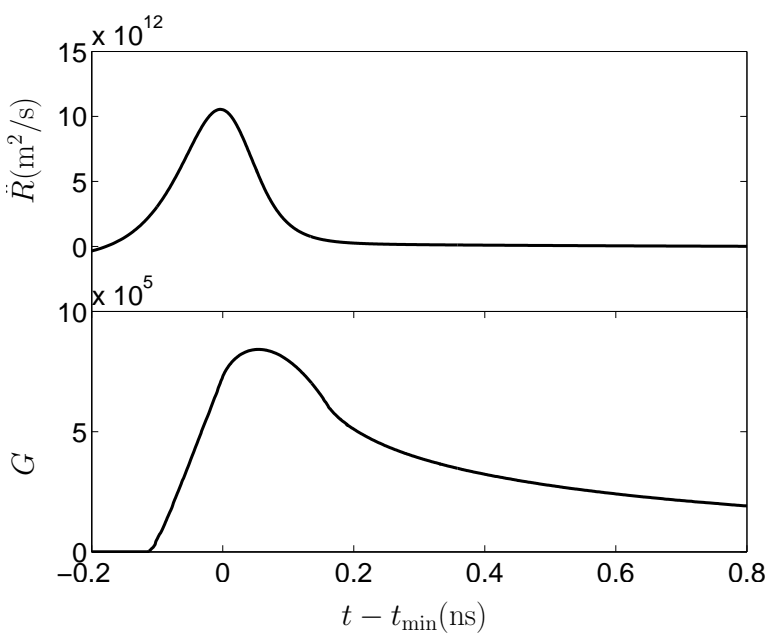

Fig. 1. (a) Time variation of the dimensional bubble wall acceleration $\ddot{R}$. (b) shape of function $G(t)$ in equation (7). The time axis is zoomed around the end of the bubble collapse.

by its ambient value and over-dots denote derivation with respect to dimensionless time $\tau$. The function $G(\tau)$ may be written as

$$
\begin{array}{r}
G(\tau)=\int_{0}^{\theta(\tau)} \frac{\ddot{R}\left(\theta^{\prime}\right)}{R^{2}\left(\theta^{\prime}\right)} \frac{d \theta^{\prime}}{\sqrt{\theta-\theta^{\prime}}}, \\
\text { with } \theta(\tau)=\int_{0}^{\tau} R^{4}(s) d s .
\end{array}
$$

Both quantities $I$ and $G$ can be evaluated easily once the bubble dynamics $R(\tau)$ is known, and depend on the amplitude and frequency of the acoustic field driving the bubble. The numerical results presented hereafter are calculated from numerical solutions of a refined bubble dynamics equation. $[5,6]$

Integral $I$ is related to the average pressure gradient at the bubble wall over one acoustic period, and function $G$ is a convolution product involving the instantaneous bubble wall acceleration. At the end of the bubble collapse, $\ddot{R}$ reaches very high positive values during a very short time (typically about 12 orders of magnitude times gravity during a few nanoseconds) and consequently $G$ is also a large positive sharp function of time. Figure 1 displays for example the bubble acceleration (figure 1.a) around the point of maximum bubble compression, and the function $G$ (figure 1.b) in the case of a $4 \mu \mathrm{m}$ ambient radius argon bubble, driven by a $26 \mathrm{kHz}$ acoustic field of amplitude 1.3 bar.

\section{Predictions}

\subsection{Qualitative overview}

We now examine the practical implications of Eq. (3) by evaluating both members of the right-handside in different cases. We focus on the case $\beta<0$, so that species $\mathrm{A}$ is heavier than the bulk liquid. Looking at the average term in expression (3), and since $\beta<0$, we have $\bar{c}<1$ so that the mixture is depleted in average at the bubble wall. Conversely, from the shape of $G$, we infer that $\Delta c$ is also a positive pulse-like function, yielding a transitory periodic over-concentration around the average value at each bubble collapse. The key feature is that the absolute value of the factor $\beta \exp \beta I$ in equation (4) first increases as $\beta$ becomes more negative, but then decreases again for large negative $\beta$. Thus, at fixed acoustic pressure, a maximum over-concentration effect is expected for a medium negative value of $\beta$. This feature is made clear in figure 2 , which shows schematically three possible behaviors:

- small $|\beta|$ (fig. (2.a): $\exp \beta I \simeq 1$. The average depletion is unnoticeable, and so do the oscillatory concentration peaks. The mixture remains almost unsegregated.

- moderate $|\beta|$ (fig. (2.b): $\exp \beta I<1$. The average depletion is noticeable but not total. But since $|\beta \exp \beta I|$ is large, the oscillatory concentration peaks reach high values. The species A will be largely over-concentrated at each bubble collapse.

- large $|\beta|$ (fig. (2.c): $\exp \beta I \ll 1$. The average depletion is almost total, but $|\beta \exp \beta I|$ decreases again toward very low values, so that the oscillatory concentration peaks drop again to low amplitudes. The oscillatory effect cannot counteract the large average depletion effect so that species A is nearly absent from the bubble wall.

Of course, since $I$ and the peak value of $G(\tau)$ are increasing functions of the amplitude of the acoustic field driving the bubble, the behavior of the same molecule or nano-particle can fall in any of the three cases described above by varying the bubble forcing. The role of the latter is made clear in the following section.

\subsection{Quantitative predictions: copper nano-particles}

We consider a solution of water with dispersed copper nano-particles as species A. In this case $\bar{V}_{A} / M_{A}$ should be replaced by the inverse of the 

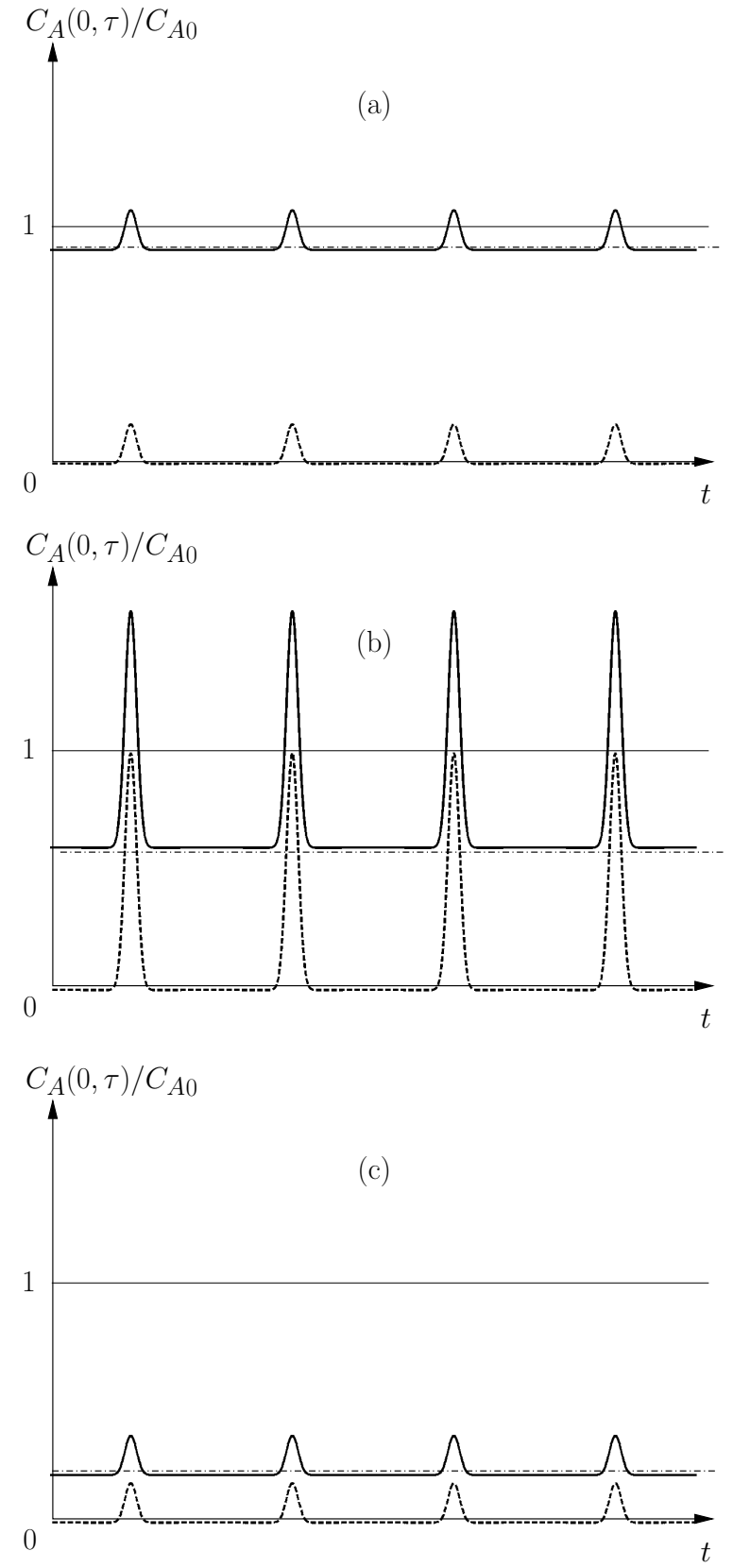

Fig. 2. Qualitative behaviors expected from equation (3) for average and peak segregation as $|\beta|$ increases (from a to c). Dashed line: oscillatory part $\Delta c(t)$; dash-dotted line: average part $\bar{c}$; solid lines: total segregation ratio $C_{A}(0, t) / C_{A 0}$. The horizontal thin line of ordinate 1 materializes an unsegregated mixture. Note that the curves are not numerical results, they are drawn for illustration purposes only. particle density $1 / \rho_{A}$ and the equivalent molar weight is obtained from the particle radius $R_{A}$ by

$$
\mathcal{N}_{a} \frac{4}{3} \pi R_{A}^{3} \rho_{A}=M_{A}
$$

where $\mathcal{N}_{a}$ is the Avogadro number. The diffusion coefficient is estimated from Stokes-Einstein theory by

$$
D=\frac{k_{B} T}{6 \pi \mu R_{A}},
$$

where $k_{B}$ the Boltzmann constant and $\mu$ the liquid viscosity. The parameter $\beta$ increases with the particle radius $R_{A}$ and the curves of figure (2) can thus be interpreted, from top to bottom, as the evolution of the segregation behavior when the nano-particles size is increased. Therefore, at the bubble wall:

- small particles remain almost unsegregated,

- medium particles are slightly depleted in average at the bubble wall, but largely over-concentrated at the end of each bubble collapse,

- large particles are always held far away from the bubble wall.

This can be clearly seen in figure 3 which displays, for various particle sizes, the average depletion (a), and the maximum of the over-concentration peaks (b), as a function of the acoustic pressure driving a $4 \mu \mathrm{m}$ argon bubble at $26.5 \mathrm{kHz}$. The average depletion (Fig. 3.a) is seen to increase both with particle size and acoustic pressure. It becomes almost total for $10 \mathrm{~nm}$ particles for an acoustic pressure slightly above 1 bar, that is to say just above the Blake threshold. Now looking at figure 3.b, it can be seen that over-concentration peak amplitude crosses a maximum as the particle size is increased, which has been explained previously from a qualitative point a view (see the dashed lines in figure 2). There is also a maximum if the acoustic pressure is varied, which can be seen clearly on the $5 \mathrm{~nm}$ and $10 \mathrm{~nm}$ curves (it would also be the case for the $1 \mathrm{~nm}$ and $2 \mathrm{~nm}$ curves if the calculations had been extended for higher acoustic pressures). The three typical cases depicted in Fig. 2(a-c) have been materialized on Fig. (3) by respectively filled circles (case a), filled squares (case b), and open circles (case c). Note that in case $\mathrm{b}$, the particles are over-concentrated by more than 2 orders of magnitude.

\subsection{Relevance to homogeneous nucleation of crystals}

Crystallization in solution belongs to the class of first-order phase transitions. Thus, before the tran- 
$\bar{c}$

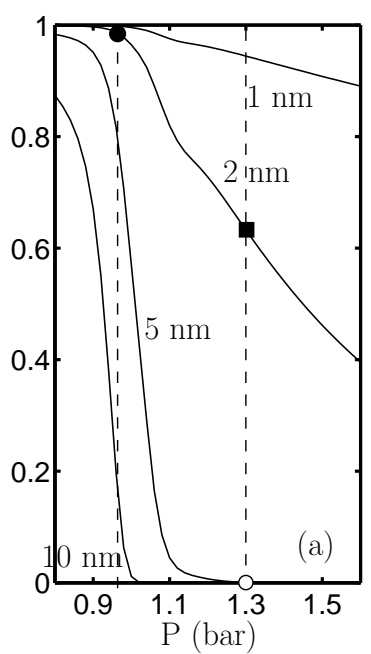

$\Delta c$

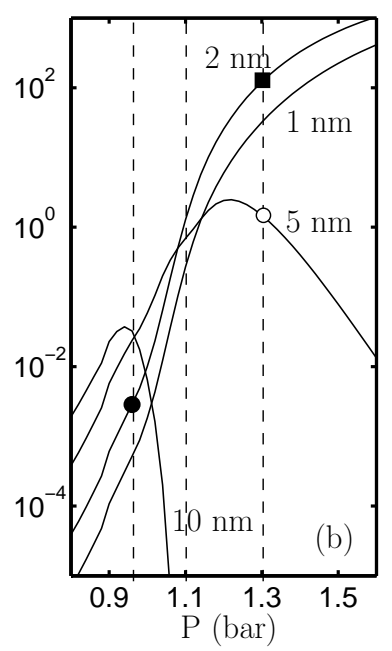

Fig. 3. Predicted segregation ratio in a mixture of water with copper nano-particles around a $4 \mu \mathrm{m}$ argon bubble driven at $26.5 \mathrm{kHz}$ : $(a)$ average segregation ratio, (b) oscillatory peak segregation ratio. On both graphs: filled circles: $2 \mathrm{~nm}$, $P=0.96$ bar; filled squares: $2 \mathrm{~nm}, P=1.3$ bar; open circles: $5 \mathrm{~nm}, P=1.3$ bar

sition occurs, the solute phase stays in a metastable equilibrium state, separated from the truly stable one by an energy barrier, that the system must cross for the first crystals to appear. Since crossing this energy barrier can only be achieved by fluctuations, a time-interval is required for the transition to take place, referred as "induction time" in the context of crystallization. Various studies demonstrate that the application of ultrasounds in the solution efficiently reduces this induction time.[7] Figure (4) shows experimental results on crystallization of potassium sulfate [8]: the induction time is displayed as the function of supersaturation for silent crystallization (open circles) and two different ultrasound powers (plus and square signs). It is seen that for medium supersaturations, the induction time can be reduced by more than one order of magnitude. But more interesting, for smaller supersaturations, insonification is a necessary condition for crystallization to occur in a reasonable time-interval.

Following classical nucleation theory [9], the lowest energy expense for the phase transition is achieved by progressive aggregation of solute molecules, referred hereafter as "monomers", to form molecular clusters. These clusters have the density of the new phase and they must reach a critical size, referred as "nucleus", for which the surface

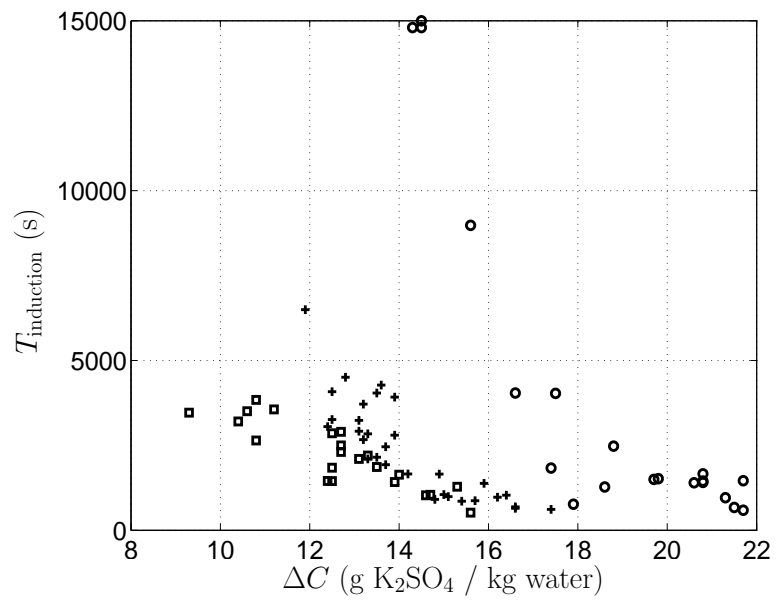

Fig. 4. (From ref. [8]) Induction time as a function of supersaturation measured for crystallization of $\mathrm{K}_{2} \mathrm{SO}_{4}$, in silent mode (circles), and in insonified mode $(20 \mathrm{kHz})$ with an input power of $40 \mathrm{~W} / \mathrm{kg}$ solution (plus signs) and $120 \mathrm{~W} / \mathrm{kg}$ solution (square signs).

energy spent in the formation of the inter-phase area is exactly balanced by the excess of chemical potential. Therefore, the nucleation time is mainly the time required for the clusters to reach this critical size, and any microscopic effect enhancing the formation of the smallest clusters may enhance notably the global nucleation kinetics.

Clusters may grow by aggregation of solute molecules one by one with existing smaller clusters (figure 5). We will label in what follows $C_{1}$ the monomers, or solute molecules, and $C_{n}$ a cluster formed by $n$ monomers, and the basic aggregation mechanism can be written as the chemical equilibrium

$$
C_{n}+C_{1} \leftrightarrow C_{n+1} .
$$

One may also consider direct aggregation of a cluster $C_{n}$ with a cluster $C_{m}, m, n \neq 1$,

$$
C_{n}+C_{m} \leftrightarrow C_{n+m} .
$$

which would be much more efficient than (11). In fact this attachment reaction is generally neglected, since small clusters are much more rarefied than solute molecules, and have therefore negligible probability to meet.

Clusters are in fact more than a conceptual tool and have been evidenced experimentally in supersaturated solutions $[10,11]$, by observing their segregation under gravity in a sedimentation column (remember that they have the density of the solid). In fact the driving force leading to their sedimenta- 


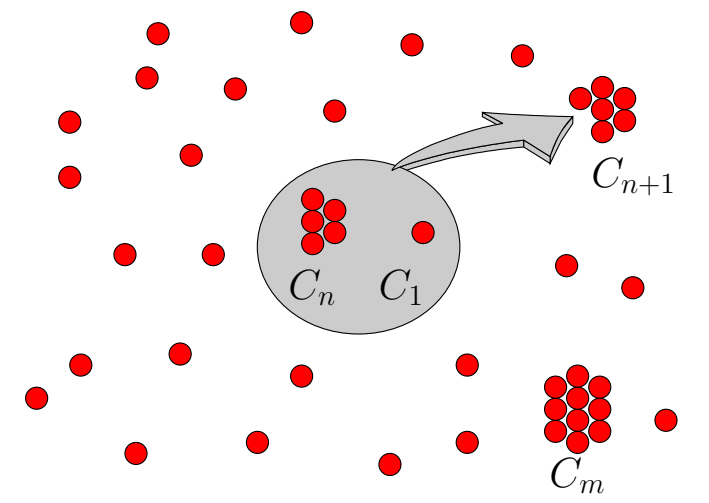

Fig. 5. Cluster growth by aggregation of a solute molecule on an existing cluster. Direct aggregation between clusters has low probability because of the low number of clusters compared to solute molecules.

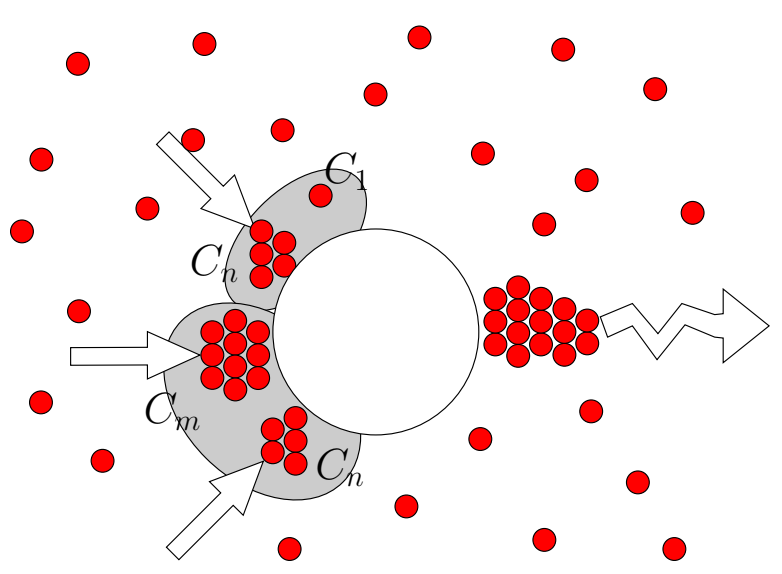

Fig. 6. Clusters growth in presence of a cavitation bubble: pressure diffusion concentrates medium clusters near the wall of the bubble at each collapse, favoring aggregation between these clusters and a solute molecule, and possibly direct aggregation between two clusters. Large clusters are then held far from the bubble by the average effect.

tion is precisely the phenomenon of pressure diffusion underlying our theory. One may therefore reasonably expect clusters to be segregated efficiently by the pressure gradients around a cavitation bubble. Keeping figure 2 in mind, the following mechanism of crystallization enhancement by a cavitation bubble is proposed, in the case where the solid nucleating phase is denser than the host liquid $(\beta<0)$ :

(i) the solute molecules and smallest clusters (figure 2.a) would be scarcely segregated at the bubble wall, either in average, or at the bubble collapse.

(ii) medium clusters (figure 2.b) would be slightly depleted in average, but strongly overconcentrated at the bubble wall at the end of the collapse (note from figure 3 that the predicted over-concentration may reach several orders of magnitude for nanometric species). This would therefore increase their probability of meeting a monomer (fig. 5), which would shift equilibrium (11) to the right. But moreover, since at the end of the collapse, the bubble wall is a meeting point for medium clusters, it may also enhance drastically the usually neglected direct aggregation mechanism (12) (fig. 6).

(iii) when they reach a large size, the cluster fall in the case of figure 2.c: their periodic overconcentration becomes small again, and their average depletion is important, so that they will be constantly held far away from the bubble (fig. 6). It is interesting to note that this fact may also contribute to the global efficiency of the cluster growth process in virtue of Le Chatelier's principle: indeed, this means that the large-size products of reactions (11), (12) would be constantly removed, thus shifting again these reactions to the right.

The bubble neighborhood may therefore act as an efficient reactor of clusters formation, which would then be released in the liquid. There arises however the following question: is the cluster attachment a sufficiently rapid process to occur on the rather short duration of the oscillatory over-concentration ? In reference [4], it was calculated that the characteristic timescale of species over-concentration, in SBSL conditions, ranges between 1 ns and $1 \mu \mathrm{s}$, and strongly decreases with the acoustic pressure. The attachment dynamics between clusters constitutes a difficult matter (see ref. [9] for an extensive discussion), because there are no direct experimental data, and one can only revert to models of the fundamental microscopic processes involved. In solutes, clusters attachment is generally considered diffusion-limited in classical crystallization conditions, and is a rather slow process. However, in the present case, pressure diffusion not only drives many clusters to the bubble wall, but it drives them with a velocity depending on their size, so that two clusters of different sizes would travel relative to each other with a nonzero velocity. This feature is expected to increase the attachment frequency and would also favor the cluster formation compared to the case of silent crystallization. The next step in theory would therefore consist in evaluating carefully the attachment frequency and solve the non-stationary global equation of cluster formation kinetics [9] taking 
into account the periodic over-concentration near the bubble wall. In the absence of such a theory for now, only tentative conclusions may be drawn. As stated above, increasing the driving level increases the transitory over-concentration, but also decreases its duration. The two behaviours may therefore compete, and one may reasonably conjecture that an optimum yield could be obtained for a moderate acoustic pressure, above which clusters would not have enough time to aggregate.

Besides, since the over-concentration process is mainly driven by the inertial motion of the bubble, its magnitude is mainly dependent on the expansion ratio $R_{\max } / R_{0}$. The latter decreases with frequency, and the transitory over-concentration level is thus expected to decrease with frequency, which is confirmed by numerical results presented in Ref. [4]. At a macroscopic level however, this may compensated by the increasing number of collapses per unit-time as frequency is increased. Such a competition between less-energetic but more numerous collapses as frequency is increased are well-known in sonochemistry [12].

The bubble lifetime has no influence on the transitory effect, since the latter occurs at the end of the bubble collapse. If the bubble undergoes its collapse until the end, the over-concentration will take place, even if the bubble breaks up afterwards. It may have however an effect on the average depletion, since, as shown in Ref. [4], the latter is a rectified effect, and progressively builds over a large number of periods (of the order of $R_{0}^{2} \omega / 2 \pi D$ ). A short-living bubble should therefore not have enough time to deplete completely its surface in the heaviest species. A similar issue arises for potential argon rectification of inertial air bubbles in a multi-bubble field [13]. Thus, case (c) in Fig. 2 may not occur for short-living bubbles, and large clusters would not be ejected far from the bubble by this mechanism. Anyway, they would probably be dispersed in the liquid by the bubble break-up.

One may finally seek an upper bound for the size of the particles covered by the present theory. This issue is motivated by the reported attraction and apparent sticking of dust or dirt particles to a levitating single bubble. Such a description suggests that the effect is cumulative, the bubble attracting gradually the particle over several oscillation periods. First, within the present theory, the particle must be lighter than water for attraction to occur in average. Secondly, the limit of the present theory is attained when the relaxation time of the particle (the time it needs to reach its terminal velocity when submitted to an acceleration field) is no longer smaller than the characteristic time-scale of the acceleration field (here the acceleration of the bubble wall). From our calculations, for particles twice lighter than water in standard levitation conditions $\left(R_{0}=4 \mu \mathrm{m}, f=\right.$ $20 \mathrm{kHz}, P=1.3$ bar), inertial effects would become important for particles radii larger than $20 \mathrm{~nm}$. It is unlikely that the reported dust particles, if visible, would have such a small size, so that they would not be covered by the present theory. It may be tentatively suggested that even with inertial effects, a cumulative attraction process could still occur, but other causes such as hydrophobic effects cannot be ruled out.

\section{Conclusion}

An inertial bubble is theoretically able to segregate large molecules or nano-particles from a host liquid of different density. A species denser than the liquid should be depleted on average at the bubble wall and largely over-concentrated during a very short time at the end of the bubble collapse. In the case where the species is a solute in a metastable equilibrium state, segregation may enhance the attachment of solute molecules to form clusters, and therefore increase the nucleation kinetics, as commonly observed in sono-crystallization. Besides, the design of a dedicated single-bubble experiment is in progress, in order to assess the effectiveness of the segregation phenomenon.

\section{References}

[1] R. B. Bird, W. E. Stewart, E. N. Lightfoot, Transport phenomena, John Wiley and sons, 1960.

[2] B. P. Barber, R. A. Hiller, R. Lofstedt, S. J. Putterman, K. R. Weninger, Defining the unknowns of sonoluminescence., Phys. Rep. 281 (1997) 65-143.

[3] B. D. Storey, A. J. Szeri, Mixture segregation within sonoluminescence bubbles, J. Fluid Mech. 396 (1999) 203-221.

[4] O. Louisnard, F. Gomez, R. Grossier, Segregation of a liquid mixture by a radially oscillating bubble, Accepted in J. Fluid Mech.

[5] B. D. Storey, A. Szeri, A reduced model of cavitation physics for use in sonochemistry, Proc. R. Soc. London, Ser. A 457 (2001) 1685-1700. 
[6] R. Toegel, B. Gompf, R. Pecha, D. Lohse, Does water vapor prevent upscaling sonoluminescence ?, Phys. Rev. Lett. 85 (15) (2000) 31653168.

[7] G. Ruecroft, D. Hipkiss, T. Ly, N. Maxted, P. W. Cains, Sonocrystallization: The use of ultrasound for improved industrial crystallization, Org. Process Res. Dev. 9 (2005) 923-932.

[8] N. Lyczko, F. Espitalier, O. Louisnard, J. Schwartzentruber, Effect of ultrasound on the induction time and the metastable zone widths of potassium sulphate., Chem. Eng. Journ. 86 (2002) 233-241.

[9] D. Kaschiev, Nucleation : Basic theory with applications, Butterworths-Heinemann, 2000.

[10] J. W. Mullin, C. L. Leci, Evidence of molecular cluster formation in supersaturated solutions of citric acid., Phil. Mag. 19 (161) (1969) 10751077.

[11] M. A. Larson, J. Garside, Solute clustering in supersaturated solutions., Chem. Eng. Sci. 41 (5) (1986) 1285-1289.

[12] C. Pétrier, A. Francony, Ultrasonic waste-water treatment: incidence of ultrasonic frequency on the rate of phenol and carbon tetrachloride degradation, Ultrasonics Sonochemistry 4 (1997) 295-300.

[13] O. Louisnard, F. Gomez, Growth by rectified diffusion of strongly acoustically forced gas bubbles in nearly saturated liquids, Phys. Rev. E 67 (036610) (2003) 1-12. 